\title{
Lymphangiomatosis of the sigmoid colon - a rare cause of lower gastrointestinal bleeding: A case report and review of the literature
}

\author{
GUIFANG LU, HONGXIA LI and YUANYUAN LI
}

Department of Gastroenterology, The First Affiliated Hospital of Xi'an Jiaotong University, Xi'an, Shaanxi 710061, P.R. China

Received March 17, 2015; Accepted July 20, 2016

DOI: $10.3892 / 01.2016 .5399$

\begin{abstract}
The present study reports the case of a 79-year-old Chinese man who presented to The First Affiliated Hospital of Xi'an Jiaotong University (Xi'an, China) for the treatment of lower gastrointestinal bleeding. Multiple cystic masses in the sigmoid colon were observed with colonoscopy, and through endoscopic ultrasound (EUS), these cystic masses were confirmed to be echo-free and to exhibit septal walls in the submucosal layer; in consequence, lymphangiomatosis of the sigmoid colon was diagnosed. Considering the repeated bleeding, laparoscopy-assisted partial sigmoid colon resection was performed. The excised specimens were multiple vesicular and soft masses of $\sim 1 \mathrm{~cm}$ in diameter, which were located in the submucosal layer and were surrounded by flat endothelial cells. Immunohistochemistry revealed that the specimens were positive for the specific lymphatic endothelial marker D2-40. The pathological diagnosis was consistent with the EUS findings. In the 2-year follow-up after the operation, no bleeding or other complications were noticed.
\end{abstract}

\section{Introduction}

Lymphangioma is an uncommon malformation of the lymphatic system (1). The majority of lymphangiomas usually occur in the head, neck regions and axillary areas, but rarely in the colon (2-4). Colonic lymphangioma used to be considered an extremely rare disease, but recently, along with the increasing prevalence of endoscopy and endoscopic ultrasound (EUS), it has become more commonly encountered and has been reported more frequently $(5,6)$. There are several reports on the use of EUS for the diagnosis of colonic lymphangioma $(4,6)$. The present study reports a case of colonic lymphangiomatosis manifested as recurrent bowel bleeding, which was diagnosed by EUS and treated with laparoscopic segmental sigmoid

Correspondence to: Professor Hongxia Li, Department of Gastroenterology, The First Affiliated Hospital of Xi'an Jiaotong University, 277 Yanta West Road, Xi'an, Shaanxi 710061, P.R. China E-mail: hongxia1105@126.com

Key words: lymphangiomatosis, sigmoid colon, bowel bleeding, case report colon resection. In addition, the relevant medical literature on colonic lymphangiomatosis is reviewed.

\section{Case report}

A 79-year-old Chinese man, who had no personal or familial history of any specific disease, presented to the People's Hospital of Hu County (Xi'an, China) in 2012 with intermittent attacks of bowel bleeding and abdominal discomforts for 3 months. Physical examination and complete blood cell count were unremarkable, with the exception of anemia. Fecal occult blood test was positive. The biochemical tests were all normal, and the levels of carcinoembryonic antigen, carbohydrate antigen (CA)125 (11.41 U/ml; normal range, 0.00-35.00 U/ml) and CA19-9 (14.34 U/ml; normal range, 0.00-39.00 U/ml) were within the normal limits. Upper abdominal ultrasound and chest X-ray displayed no specific findings. Colonoscopy revealed multiple cystic masses with a translucent and smooth surface, ranging from 4 to $8 \mathrm{~mm}$ in diameter, located in the sigmoid colon. The color and surface characteristics of the lesions had no difference with those of the surrounding normal mucosa, and no ulcerations or erosions were present (Fig. 1). By EUS, these cystic masses were confirmed to be echo-free and to exhibit septal walls in the submucosal layer (Fig. 2).

Based on the colonoscopy and EUS findings, the lesions were diagnosed as lymphangiomatosis of the sigmoid colon. Considering the repeated bleeding, laparoscopy-assisted partial sigmoid colon resection was performed. Surgical findings were multiple bulges on the serosal surface of the sigmoid colon by laparoscopy. The excised specimens were multiple masses of $\sim 1 \mathrm{~cm}$ in diameter, which were vesicular and soft. Histological examination revealed that the cysts were located in the submucosal layer and were surrounded by flat endothelial cells. Immunohistochemistry was positive for D2-40 (ready-to-use anti-D2-40; MAB-0567; MXB, Fuzhou, China), a specific lymphatic endothelial marker (Fig. 3). The pathological diagnosis was submucosal cystic lymphangioma of the sigmoid colon. In the 2-year follow-up after the operation, no bleeding or other complications were noticed.

\section{Discussion}

Lymphangiomas are usually detected in the head, neck and axillary areas, which account for $50-75 \%$ of all cases, while 

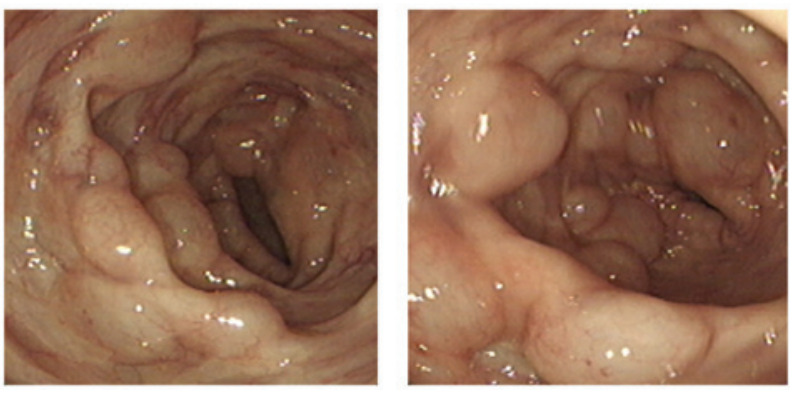

Figure 1. Colonoscopy findings: Multiple cystic masses ranging from 4 to $8 \mathrm{~mm}$ in diameter were located in the sigmoid colon, and displayed a translucent and smooth surface, similarly to that of the surrounding normal mucosa.
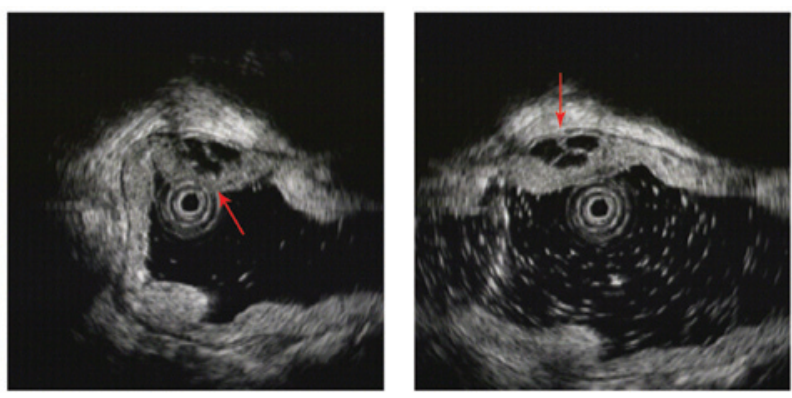

Figure 2. EUS images obtained using an EUS probe. The cystic masses observed (red arrows) were confirmed to be echo-free and to exhibit septal walls in the submucosal layer. EUS, endoscopic ultrasound.

only 5\% of cases occur in the abdomen (2-4). Intra-abdominal lymphangiomas frequently locate in the mesentery, greater omentum and retroperitoneum, but rarely in the colon (7). In 1932, Chisholm and Hillkowitz reported the first case of rectal lymphangioma (8). For a long time, colonic lymphangioma has been regarded as an exceptionally rare illness (5).

With the widespread use of colonoscopy, an increased number of colonic lymphangiomas have been detected (5). Although the specific mechanisms contributing to colonic lymphangioma are unclear, the acknowledged main cause is a congenital malformation of the lymphatic system, which results in an abnormal dilatation and proliferation of the lymphatic channel, leading to the formation of cystic masses $(9,10)$. The reasons leading to secondary colonic lymphangioma include abdominal trauma, partial lymphatic obstruction and inflammation (11-13). According to Matsuda et al (5), the age distribution of the patients with colonic lymphangioma is 1-83 years, and the incidence is higher in males, with a gender ratio of 2-2.5:1 in Japan. Regarding site, the majority of colonic lymphangioma cases occur in the transverse colon (5). In the present case, the patient was a 79-year-old male, and the lesions were discovered in the sigmoid colon.

The clinical signs and symptoms of colonic lymphangioma vary depending on the size and position of the lesion, and include abdominal discomfort such as abdominal pain and abdominal distension, colonic bleeding, acute abdomen and protein-losing enteropathy $(5,14,15)$. The current patient presented with intermittent attacks of colonic bleeding. The diagnosis of colonic lymphangioma depends on X-ray and endoscopic examination, and EUS provides a novel
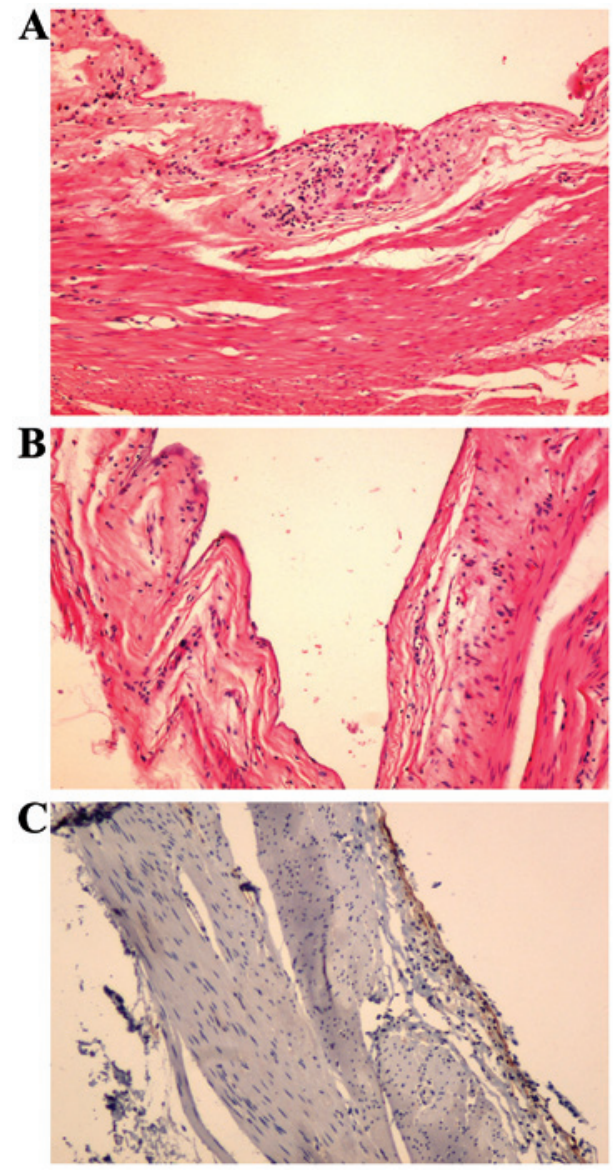

Figure 3. Immunohistochemical staining. (A) H\&E staining of dilated lymphatic vessels constituted by the fibrous cystic walls, where few lymphocytes were present (magnification, x100). (B) H\&E staining of dilated lymphatic vessels (magnification, x200). (C) Immunohistochemical D2-40 expression (brown color) in a dilated lymphatic vessel (magnification, x100). H\&E, hematoxylin and eosin.

and powerful method for the diagnosis of colonic lymphangioma (5). EUS aids to display the entire hierarchy of the colon wall and the association between the lesion and the colon wall; in addition, it aids to ascertain whether the lesion is separated, and to determine the nature of the lesion according to the echo characteristics (16). The majority of colonic lymphangiomas are isoechoic or hypoechoic, and pathological examination is the gold standard for diagnosis $(5,16,17)$. The lesions detected in colonic lymphangioma cases result from malformations of the lymphatic tissue, causing an abnormal dilatation and mass-like proliferation of lymphatic channels, which enables to observe the submucosal lymphatic cysts and fiber-like separation by hematoxylin and eosin staining $(16,17)$. A history of recurrent bowel bleeding and anemia is an important clue that should alert clinicians about the possibility of colonic lymphangioma.

Regarding therapy, since colonic lymphangioma is benign, $\sim 10 \%$ of cases ease naturally $(2,18)$, Lee et al (19) recommended regular follow-ups rather than treatment for those asymptomatic colonic lymphangiomas. Positive treatments include surgery, endoscopic resection and drug injection. Complete surgical resection is the first choice for large lymphangiomas accompanied with complications such as bowel obstruction, bleeding, volvulus or intussusception (5). With the development of endoscopy, endoscopic resection is 
preferred for pedunculated and semipedunculated lesions of $<2 \mathrm{~cm}$ (20). In the present case report, the patient exhibited recurrent bowel bleeding, and his lesions measured $\leq 10 \mathrm{~cm}$; thus, laparoscopy-assisted surgical resection was conducted. During 2 years of follow-up after the operation, no bleeding or other complications were noticed.

Colonic lymphangioma is a rare disease. The present study reports a case of colonic lymphangioma that presented with recurrent bowel bleeding and anemia, which revealed that lymphangiomas may be a cause of gastrointestinal bleeding.

\section{References}

1. Watanabe T, Kato K, Sugitani M, Hasunuma O, Sawada T, Hoshino N, Kaneda N, Kawamura F, Arakawa Y and Hirota T: A case of multiple lymphangiomas of the colon suggesting colonic lymphangiomatosis. Gastrointest Endosc 52: 781-784, 2000.

2. Alqahtani A, Nguyen L, Flageole H, Shaw K and Laberge JM: 25 years' experience with lymphangiomas in children. J Pediatr Surg 34: 1164-1168, 1999.

3. Chung JH, Suh YL, Park IA, Jang JJ, Chi JG, Kim YI and Kim WH: A pathologic study of abdominal lymphangiomas. J Korean Med Sci 14: 257-262, 1999.

4. Zhuo CH, Shi DB, Ying MG, Cheng YF, Wang YW, Zhang WM, Cai SJ and Li XX: Laparoscopic segmental colectomy for colonic lymphangiomas: A definitive, minimally invasive surgical option. World J Gastroenterol 20: 8745-8750, 2014.

5. Matsuda T, Matsutani T, Tsuchiya Y, Okihama Y, Egami K, Yoshioka M, Maeda S and Onda M: A clinical evaluation of lymphangioma of the large intestine: A case presentation of lymphangioma of the descending colon and a review of 279 Japanese cases. J Nippon Med Sch 68: 262-265, 2001.

6. Jung SW, Cha JM, Lee JI, Joo KR, Choe JW, Shin HP and Kim KY: A case report with lymphangiomatosis of the colon. J Korean Med Sci 25: 155-158, 2010.

7. Roisman I, Manny J, Fields S and Shiloni E: Intra-abdominal lymphangioma. Br J Surg 76: 485-489, 1989.
8. Chisholm AJ and Hillkowitz P: Lymphangioma of the rectum. The American Journal of Surgery 17: 281-282, 1932.

9. de Perrot M, Rostan O, Morel P and Le Coultre C: Abdominal lymphangioma in adults and children. Br J Surg 85: 395-397, 1998.

10. Weeda VB, Booij KA and Aronson DC: Mesenteric cystic lymphangioma: A congenital and an acquired anomaly? Two cases and a review of the literature. J Pediatr Surg 43: 1206-1208, 2008.

11. Kim JH, Ryu WS, Min BW, Song TJ, Son GS, Kim SJ, Kim YS and Um JW: Acquired omental cystic lymphangioma after subtotal gastrectomy: A case report. J Korean Med Sci 24: 1212-1215, 2009.

12. Tezuka K, Ogawa Y, Satake K, Ohira M, Yamada S, Uno H, Wakasa $\mathrm{K}$ and Hirakawa K: Lymphangioma of the lesser omentum associated with abdominal esophageal carcinoma: Report of a case. Surg Today 32: 362-366, 2002.

13. Fisher D and Hiller N: Case report: Giant tuberculous cystic lymphangioma of posterior mediastinum, retroperitoneum and groin. Clin Radiol 49: 215-216, 1994.

14. Matsuba Y, Mizuiri H, Murata T and Niimi K: Adult intussusception due to lymphangioma of the colon. J Gastroenterol 38: 181-185, 2003.

15. Kim J, Han D, Hong CH, Lee HL, Kim JP, Sohn JH and Hahm JS: Colonic lymphangiomatosis associated with protein-losing enteropathy. Dig Dis Sci 50: 1747-1753, 2005.

16. Black T, Guy CD and Burbridge RA: Retroperitoneal cystic lymphangioma diagnosed by endoscopic ultrasound-guided fine needle aspiration. Clin Endosc 46: 595-597, 2013.

17. Gottlieb K and Elkharwily A: Endoscopic ultrasound evaluation of a cystic lymphangioma of the colon. J Ultrasound Med 26: 1803-1804, 2007.

18. Steyaert H, Guitard J, Moscovici J, Juricic M, Vaysse P and Juskiewenski S: Abdominal cystic lymphangioma in children: Benign lesions that can have a proliferative course. J Pediatr Surg 31: 677-680, 1996.

19. Lee JM, Chung WC, Lee KM, Paik CN, Kim YJ, Lee BI, Cho YS and Choi HJ: Spontaneous resolution of multiple lymphangiomas of the colon: A case report. World J Gastroenterol 17: 1515-1518, 2011.

20. Sato K, Maekawa T, Yabuki K, Tomita N, Eguchi M, Matsumoto M and Sugiyama N: Cystic lymphangiomas of the colon. J Gastroenterol 34: 520-524, 1999. 